\title{
A Reported Case of an Acute Shock Caused by a Mitral Prosthetic Valve Thrombosis
}

\author{
Ahchouch S*, Ballouk R, Mounir R, Ait Kajjat O, Malki M, Boumaaz M, Zaimi A, Loudiyi N, Asfalou I, Raissouni M,
} Benyass A

Centre de Cardiologie de l'Hôpital Militaire d'Instruction Mohamed V

DOI: $10.36348 /$ sjmps.2020.v06i02.016 $\quad$ | Received: 18.02 .2020 | Accepted: 25.02 .2020 | Published: 29.02 .2020

*Corresponding author: S. Ahchouch

\section{Abstract}

Introduction: Mitral prosthetic thrombosis (MPV) is a rare but dangerous complication. It is suspected by clinical examination; the diagnosis of MPV is made by trans-thoracic echocardiography and held by the trans-esophageal echocardiography; or a fluoroscopy which shows a lack of prosthesis opening. The treatment is based on the early establishment of anti-coagulation and surgical cure. The thrombolysis is an alternative therapy. Case report: We report the case of a 47-year-old man who undergone a mitroaortic mechanical prosthesis replacement 6 years ago. However, since a year, his INR has been sub therapeutic. He presented a 5 days history of a chest pain and dyspnea. A passing at the intensive care diagnosed a shock according to the cool extremities, sweats, tachypnea and low blood pressure. The ECG showed atrial fibrillation with complete left bundle block. An emergency TTE was performed and revealed a MPV with high transvalvular gradient, while the aortic prosthesis was functional. The left ventricle was dilated and globally hypo contractile; TEE was emergently performed and showed à stuck leaflet caused by thrombus with associated with a thrombus at the left auricle. Patient's hemodynamic condition required a surgical cure with thrombectomy of the left atrium. Conclusions: Through this case we highlight the interest of echocardiography to diagnose the etiology of shock, and the importance of early management to avoid any complications.

Keywords: Mitral prosthetic, clinical examination, echocardiography thrombectomy mitroaortic.

Copyright @ 2020: This is an open-access article distributed under the terms of the Creative Commons Attribution license which permits unrestricted use, distribution, and reproduction in any medium for non-commercial use (NonCommercial, or CC-BY-NC) provided the original author and source are credited.

\section{INTRODUCTION}

La thrombose de prothèse est une complication grave; en Effet l'implantation endo-vasculaire d'un corps étranger avec sa surface non endothélialisée active le mécanisme d'anti-agrégation aboutissant de ce fait à la constitution d'un thrombus intra-cavitaire.

C'est une complication grevé d'une mortalité et d'une morbidité élevées et nécessitant un traitement urgent. Nous rapportons le cas d'une thrombose de prothèse mécanique mitrale aigue compliquée de choc cardiogénique, traitée chirurgicalement avec bonne évolution.

\section{Cas Clinique}

Nous rapportons le cas d'un patient âgé de 47ans sans facteurs de risque cardiovasculaires, ayant bénéficié 6 ans plutôt d'un double remplacement valvulaire mitral et aortique par prothèse mécanique de type Sorin (respectivement $\mathrm{N}^{\circ} 29$ et 23 ) pour affection rhumatismale fuyante. Depuis le patient était asymptomatique. Notons toutefois que le patient présentait un INR en dessous de la fourchette thérapeutique depuis lan par mauvaise observance thérapeutique.

La symptomatologie actuelle remontait à cinq jours avant son admission par l'aggravation rapide d'une dyspnée de base passant du stade II au Stade IV de la NYHA (New York Heart Association) motivant la consultation aux urgences. L'examen clinique réalisé alors, avait retrouvé un patient conscient mais pâle, polypnéique en sueurs. Le diagnostic de choc cardiogénique était retenu devant une hypotension à $87 / 55 \mathrm{mmHg}$ avec extrémités froides. L'examen cardiovasculaire notait un pouls irrégulier à $123 \mathrm{cpm}$, des bruits de prothèses étaient perçus légèrement diminués avec des signes d'insuffisance cardiaque globale. L'électrogramme effectué inscrivait une fibrillation auriculaire avec bloc de branche complet. La radiographie du thorax montrait une cardiomégalie avec ICT à 0,66, un aspect en double contours de l'arc 
inférieur droit avec surcharge hilaire bilatérale. Le bilan biologique retrouvait un INR sub-thérapeutique à 1, 33

L'échocardiographie trans-thoracique avait montré un élément mobile au niveau de la prothèse mitrale évoquant un thrombus avec accélération du flux trans-prothétique (figure 1, 2) donnant un gradient moyen à $18 \mathrm{mmHg}$ (Figure 3 ). Le ventricule gauche était dilaté en dysfonction systolique sévère (FEVG à $25 \%$ ), la prothèse aortique était de bon fonctionnement;
L'ETO avait permis de mieux visualiser un thrombus tapissant la prothèse mécanique à l'origine d'un blocage d'ailette.

Devant l'instabilité hémodynamique, une cure chirurgicale en urgence a été décidée avec mise en place d'une nouvelle prothèse de type sorin $\mathrm{N}^{\circ} 29$ (Figure 5) et thrombectomie de 1 oreillette gauche. Les suites opératoires étaient simples.
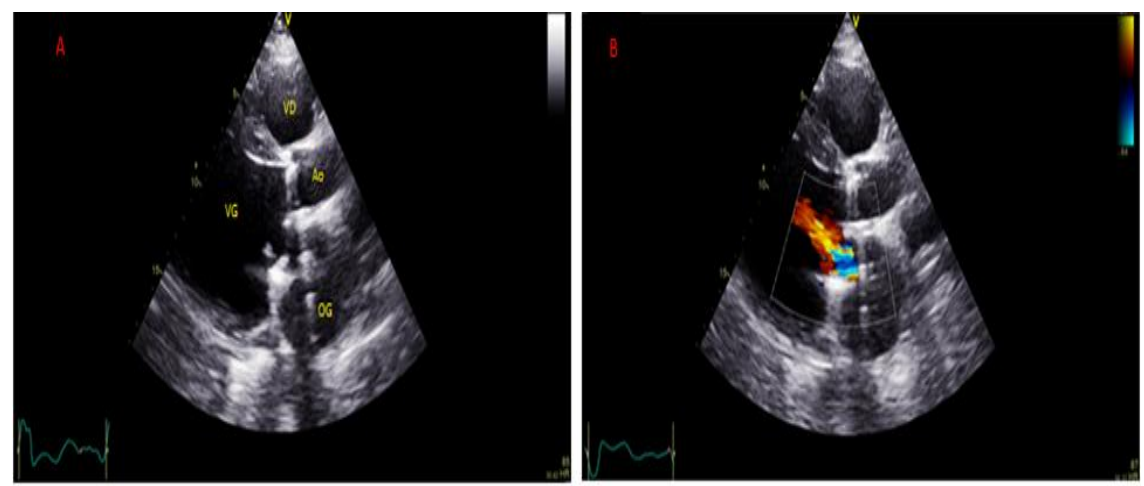

Fig-1: Coupe grand axe montrant un thrombus au niveau de la prothèse mitrale (A) avec accélération du Flux (B)
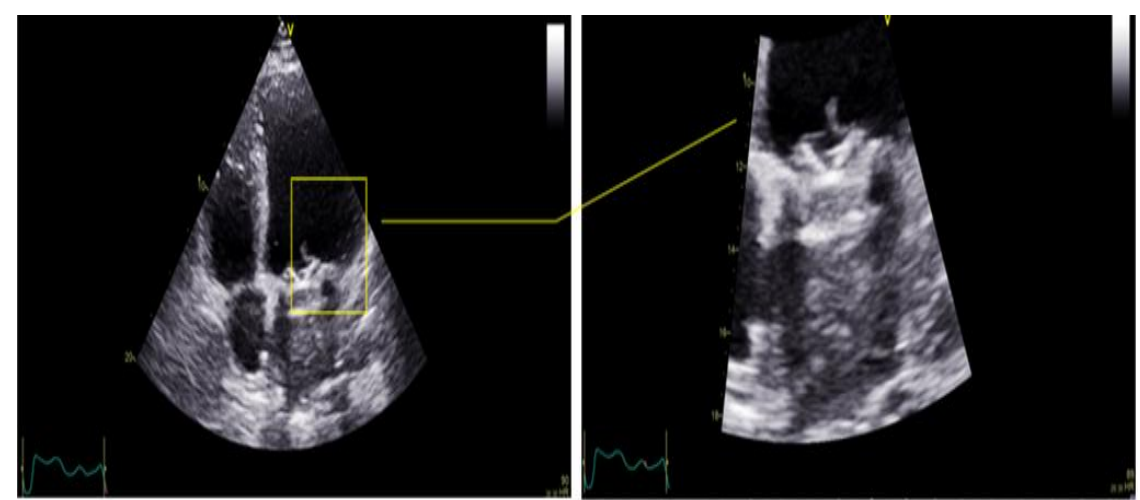

Fig-2: Coupe 4 cavités montrant une ailette bloquée avec thrombus

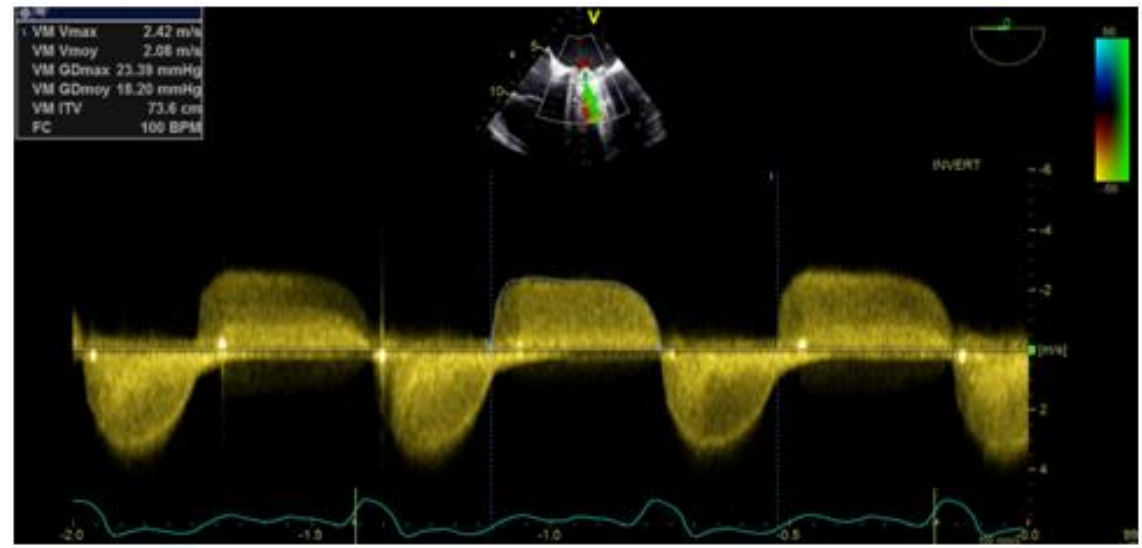

Fig-1: Gradient moyen trans-prothétique en faveur d'une sténose de la prothèse mitrale 

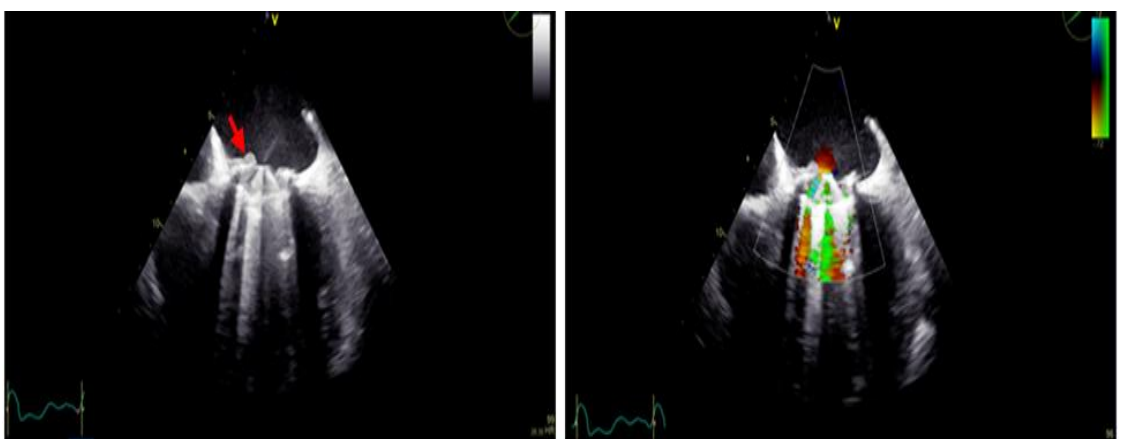

Figure 4 ETO montrant un thrombus au niveau de la prothèse mitrale (flèche rouge) avec accélération du flux trans-mitral au doppler couleur

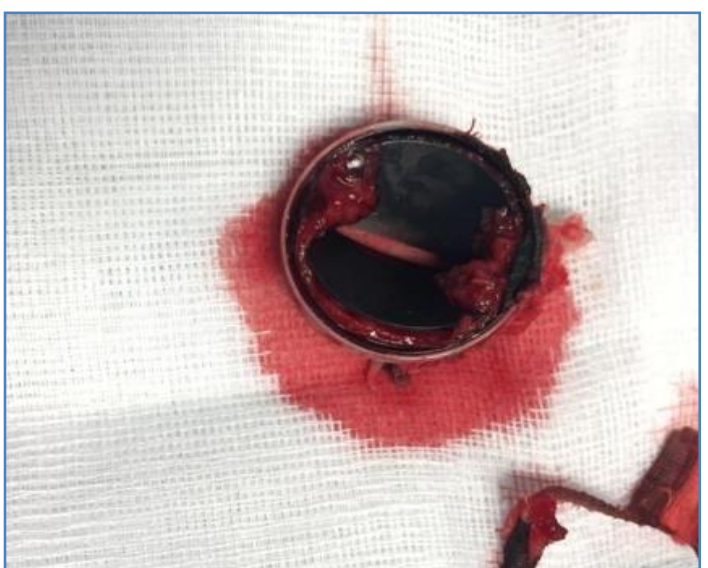

Fig-2: Thrombus au niveau de la prothèse mitrale

\section{DISCUSSION}

L'incidence annuel de la thrombose de prothèse dans les pays en voies de développement 6 , $1 \%$ contre seulement 0,3 à 1,3 patient par an dans les pays développées[1];

On en décrit plusieurs facteurs de risque de thrombose à savoir le type de valve et sa position, la présence d'une dysfonction ventriculaire, un rythme en fibrillation auriculaire...Toutefois l'anti-coagulation inadéquate en reste le facteur prédisposant le plus important [2, 3]. En effet, les patients ne présenteraient un INR à la cible que dans la moitié du temps [6]. Notre patient était à très haut risque de thrombose de prothèse. L'INR cible pour notre devrait se situer dans une fourchette de [3-5] selon les recommandations. La prévention de ces affections doit passer par une surveillance rigoureuse de l'anticoagulation.

\section{L'intérêt de l'auto-surveillance} d'anticoagulants oraux a été bien mise en évidence dans le suivi des patients porteurs de prothèses valvaires mécaniques [7, 8]. Bussey et al. avaient montré que les patients' pratiquant l'auto-surveillance hebdomadaire (PST) obtenaient une meilleur efficacité thérapeutique: $56 \%$ des INR à la cible thérapeutique passant à $81 \%$ après auto-surveillance [9].

Le diagnostic de thrombose de prothèse est soulevé devant l'installation d'une dyspnée aigue ou d'une instabilité hémodynamique. L'échocardiographie, du fait de sa disponibilité, est la méthode de choix pour le diagnostic positif et de gravité : elle permet de mettre en évidence une dysfonction de prothèse d'en apprécier la sévérité [10-11]. L'Echographie trans-oesophagienne (ETO) offre en plus une meilleure visualisation de la prothèse et permet de donner plus de précision quant à la nature de l'obstacle [12-16 ] .

La prise en charge de la thrombose de prothèse demeure controversée et suscite toujours débat: il n'y a pas d'études randomisées comparant le traitement chirurgical et la fibrinolyse, seuls des séries monocentriques, des données de registres et quelques cas ont été rapportés par la littérature.

Les sociétés savantes recommandent le traitement chirurgical en première intension devant toute thrombose de prothèse $d u$ cœur gauche avec instabilité hémodynamiques. La fibrinolyse est réservée en deuxième intension ligne pour les patients ayant un risque chirurgical élevé, hémorragique bas et chez les patient présentant une thrombose de prothèse du cœur droit[17, 18 ].

\section{CONCLUSION}

La thrombose de prothèse est une complication grave. Notre cas souligne l'importance d'un diagnostic précoce et d'un traitement rapide afin d'éviter toute complications. La cures chirurgicale est le traitement de choix.

\section{REFFERENCE}

1. Nishimura, R.A., Otto, C.M., Bonow, R.O., Et, A.L. (2014). AHA/ACC guideline for the management of patients with valvular heart disease: executive summary. A report of the American College of Cardiology/American Heart Association Task Force on Practice Guidelines. J Am Coll Cardiol, 63:2438-2488.

2. Deviri, E., Sareli, P., Wisenbaugh, T., \& Cronje, S. L. (1991). Obstruction of mechanical heart valve prostheses: clinical aspects and surgical management. Journal of the American College of Cardiology, 17(3), 646-650. 
3. Dürrleman, N., Pellerin, M., Bouchard, D., Hébert, Y., Cartier, R., Perrault, L. P., ... \& Carrier, M. (2004). Prosthetic valve thrombosis: twenty-year experience at the Montreal Heart Institute. The Journal of Thoracic and Cardiovascular Surgery, 127(5), 1388-1392.

4. Butchart, E. G., Payne, N., Li, H. H., Buchan, K., Mandana, K., \& Grunkemeier, G. L. (2002). Better anticoagulation control improves survival after valve replacement. The Journal of Thoracic and Cardiovascular Surgery, 123(4), 715-723.

5. Butchart, E. G. (1992). Thrombogenicity, thrombosis and embolism. Thrombosis, Embolism, and Bleeding. London: ICR Publishers, 172-205.

6. Baker, W. L., Cios, D. A., Sander, S. D., \& Coleman, C. I. (2009). Meta-analysis to assess the quality of warfarin control in atrial fibrillation patients in the United States. Journal of Managed Care Pharmacy, 15(3), 244-252.

7. Butchart, E. G., Payne, N., Li, H. H., Buchan, K., Mandana, K., \& Grunkemeier, G. L. (2002). Better anticoagulation control improves survival after valve replacement. The Journal of Thoracic and Cardiovascular Surgery, 123(4), 715-723.

8. Körtke, H., \& Körfer, R. (2001). International normalized ratio self-management after mechanical heart valve replacement: is an early start advantageous?. The Annals of thoracic surgery, 72(1), 44-48.

9. Bussey, H. I., Bussey, M., Bussey- Smith, K. L., \& Frei, C. R. (2013). Evaluation of Warfarin Management with International Normalized Ratio Self- Testing and Online Remote Monitoring and Management Plus Low- Dose Vitamin K with Genomic Considerations: A Pilot Study. Pharmacotherapy: The Journal of Human Pharmacology and Drug Therapy,33(11), 11361146.

10. Zoghbi, W. A., Chambers, J. B., Dumesnil, J. G., Foster, E., Gottdiener, J. S., Grayburn, P. A., ... \& Nakatani, S. (2009). Recommendations for evaluation of prosthetic valves with echocardiography and Doppler ultrasound: a report from the American Society of Echocardiography's Guidelines and Standards Committee and the Task Force on Prosthetic Valves, developed in conjunction with the American College of Cardiology Cardiovascular Imaging Committee, Cardiac Imaging Committee of the American Heart Association, the European Association of Echocardiography, a registered branch of the European Society of Cardiology, the Japanese .... Journal of the American Society of Echocardiography, 22(9), 975-1014.

11. Lancellotti, P., Pibarot, P., Chambers, J., Edvardsen, T., Delgado, V., Dulgheru, R., ... \& Magne, J. (2016). Recommendations for the imaging assessment of prosthetic heart valves: a report from the European Association of Cardiovascular Imaging endorsed by the Chinese Society of Echocardiography, the Inter-American Society of Echocardiography, and the Brazilian Department of Cardiovascular Imaging. European Heart Journal-Cardiovascular Imaging, 17(6), 589-590.

12. Özkan, M., Gündüz, S., Biteker, M., Astarcioglu, M. A., Çevik, C., Kaynak, E., ... \& Karavelioğlu, Y. (2013). Comparison of different TEE-guided thrombolytic regimens for prosthetic valve thrombosis: the TROIA trial. JACC: Cardiovascular Imaging, 6(2), 206-216.

13. Biteker, M., Duran, N. E., Gündüz, S., Gökdeniz, T., Kaya, H., Aykan, A. Ç., \& Özkan, M. (2009). Thrombolysis of an acute prosthetic mitral valve thrombosis presented with cardiogenic shock under the guidance of continuous transoesophageal monitoring. European Journal of Echocardiography, 10(3), 468-470.

14. Girard, S. E., Miller, F. A., Orszulak, T. A., Mullany, C. J., Montgomery, S., Edwards, W. D., ... \& Tajik, A. J. (2001). Reoperation for prosthetic aortic valve obstruction in the era of echocardiography: trends in diagnostic testing and comparison with surgical findings. Journal of the American College of Cardiology, 37(2), 579-584.

15. Barbetseas, J., Nagueh, S. F., Pitsavos, C., Toutouzas, P. K., Quiñones, M. A., \& Zoghbi, W. A. (1998). Differentiating thrombus from pannus formation in obstructed mechanical prosthetic valves: an evaluation of clinical, transthoracic and transesophageal echocardiographic parameters. Journal of the American College of Cardiology, 32(5), 1410-1417.

16. Tong, A. T., Roudaut, R., Ozkan, M., Sagie, A., Shahid, M. S., Pontes Júnior, S. C., \& Thadhani, R. (2004). Prosthetic Valve Thrombolysis-Role of Transesophageal Echocardiography (PRO-TEE) Registry Investigators. Transesophageal echocardiography improves risk assessment of thrombolysis of prosthetic valve thrombosis: results of the international PRO-TEE registry. $\mathrm{J} \mathrm{Am}$ Coll Cardiol, 43(1), 77-84.

17. Baumgartner, H., Falk, V., Bax, J. J., De Bonis, M., Hamm, C., Holm, P. J., \& Rosenhek, R. (2017). 2017 ESC/EACTS guidelines for the management of valvular heart disease. European heart journal, 38(36), 2739-2791.

18. Rick, A., Nishimur, A., Catherine, M., Otto, R.O. (2017). Bonow, Blase A. Carabello, John P. Erwiniii, lee A. Fleisher, 2017 aha/acc Focused Update of the 2014 AHA/ACC Guideline for the Management of Patients With Valvular Heart Disease: A Report of the American College of Cardiology/American Heart Association Task Force on Clinical Practice Guidelines 15 Mar 2017Circulation,135:e1159-e1195. 